\title{
La représentation du policier dans les téléfilms Ivoiriens
}

\author{
Mathieu A. Akadje \\ UFR Criminologie, \\ Université Félix Houphouët-Boigny
}

doi: 10.19044/esj.2016.v12n20p232 URL:http://dx.doi.org/10.19044/esj.2016.v12n20p232

\section{Summary}

Studying the representation of the policeman in television movies, this is partly to describe the symbolical state of the public force in the Ivorian society. This symbolic goes by the embodiment of the police function through the image of a representative of the order, acting within the framework of the police institution. This study aims at identifying the essential features of the filmic representation of the policeman and get interested in the way the society relates itself its police. The qualitative method and content analysis helped to highlight the delinquency of the policeman by filmmakers. This filmic representation of the policeman appears as a mirror of the social changes that stirs the Ivorian society.

Keywords: Policeman, television movie, racketeering, corruption, police violence

\section{Résumé}

Etudier la représentation du policier dans les téléfilms, c'est en partie décrire l'état symbolique de la force publique dans la société ivoirienne. Cette symbolique passe par l'incarnation de la fonction policière à travers la figure d'un représentant de l'ordre, agissant dans le cadre de l'institution policière. Cette étude a pour objectif de dégager les traits essentiels de la représentation filmique du policier et de s'intéresser à la manière dont une société se raconte sa police.La méthode qualitativeet l'analyse du contenu ont permis de mettre en évidence la délinquance du policier par les réalisateurs.Cette représentation filmique du policier apparait comme un miroir des changements sociaux qui agitent la société ivoirienne.

Mots-clés : Policier, téléfilm, racket, corruption, violence policière 


\section{Introduction}

Dans les sociétés, il existe des mécanismes de contrôle social institutionnalisé (Loubet, 2001) dont le rôle est d' " assurer la conformité des conduites aux normes établies dans une collectivité donnée, pour sauvegarder entre les membres de cette collectivité le dénominateur commun nécessaire à son fonctionnement » (Rocher, 1968). Parmi les organes et les institutions spécialisées chargés du maintien de l'ordre et de la sécurité figure la police. Ce terme désigne une institution, un groupe social, remplissant donc une certaine fonction par des mesures de prévention, mais disposant aussi, si nécessaire, en ultime recours, de la possibilité d'utiliser la force par la répression. Elle est une administration, une force publique qui veille à l'observation des règlements, maintenant la sécurité publique.

La police est née avec la cité. Elle est la plus ancienne des institutions de protection sociale. La police participe à l'application des règles établies dans une société. Il existe des équations telles que «Police = Sécurité» et « Plus de Police = Plus de Sécurité » (Monet, 1993) pour traduire l’influence qu'a la police sur la sécurité des citoyens. La police tend à détenir le monopole de tout ce qui touche de près ou de loin au contrôle de la criminalité. La police vise non seulement à contenir et à réduire la criminalité mais également à diminuer la peur du crime et à accroître le sentiment réel de sécurité dans la société. Etant donné l’importance de la question sécuritaire et des agents en charge de cette sécurité, on peut s’interroger sur leur représentation dans les médias.

La manière dont est représentée le policier ou la police dans les médias audiovisuels, plus particulièrement dans les téléfilms, ne peut être sans incidence sur la représentation que s'en font les consommateurs de ces films. Les scénaristes s’inspirent des évènements réels de la vie en société. La représentation de la police fait l'objet de nombreux travaux et de nombreux articles dans les médias. Par exemples, l'étude de Philippe (1999) s'attache à dégager la signification socio-politique de la mise en représentation de la police et de l'action policière. Loubet (2006) quant à luifait une étude méthodique et comparative sur la place de la police dans l'organisation politique des sociétés. Par contre, Chablon \& Léonard (2007), à travers les problèmes d'insécurité en milieu, s'attache à dégager les orientations concrètes sur la façon dont les questions se posent et sur la manière de les résoudre. Yébouet (2007) explique que le racket policier a pris de l'ampleur et constitue un handicap principal de l'institution policière. Une meilleure appréhension du phénomène permettra d'envisager des solutions. Enfin, dans un autre ouvrage, Loubet (2012) met l'accent sur l'institution policière et son rôle dans l'évolution des processus de contrôle social. 
L’étude de Philippe (1999) a servi de guide pour ce travail. Il montre quele film policier se caractérise par la rencontre de trois acteurs qui sont l'agresseur (le délinquant), la victime et le défenseur de l'ordre. La raison qui les rassemble, c'est la transgression de la norme sociale. Le délinquant, c'est l'auteur de l'action criminelle, en l'occurrence celui qui met tout en œuvre ou ceux qui mettent en commun des moyens pour réaliser une entreprise délictueuse. Pour être considéré comme délinquant il faut avoir commis une infraction, c'est-à-dire non pas seulement un fait dommageable, mais une transgression prévue précisément par la loi. Cette transgression, le policier vise à l'empêcher par des activités de prévention autant que de répression afin de contribuer à améliorer la qualité de vie des citoyens.

Délinquants et policiers fonctionnent selon une même logique. Les premiers s'en prennent aux biens de la société et les seconds s'en prennent à celui ou à ceux qui s’attaquent aux personnes et aux biens. Il arrive souvent que le policier se trouve dans des situations dans lesquelles le flic et le voyou sont indiscernables l'un de l'autre. Cette proximité entre les représentants de l'ordre et les délinquants est véritablement une constance à travers la production cinématographique (Philippe, 1999). Cependant, il est paradoxal de voir ceux qui sont chargés de faire appliquer les lois être les premiers à les transgresser. Racket, corruption, agressions (violence illégitime)...sont les actes délictueux commis par des défenseurs de l'ordre que sont censés être les policiers. La mise en représentation filmique du policier apparaît alors comme un miroir des changements sociaux. Ces téléfilms ont suscité des questionnements sur les pratiques policières. L'observation documentaire et l'analyse de contenu des films ont permis de dégager trois formes de déviances policières : le policier racketteur, le policier ripou et le policier violent. Vu la diversité des supports médiatiques mettant en représentation la police, notre choix s’est porté sur les téléfilms ivoiriens. Etudier la représentation du policier dans les téléfilms ivoiriens vise à contribuer à la connaissance de cet objet. Etudier la représentation du policier dans les téléfilms, c'est en partie décrire l'état symbolique de la force publique dans la société ivoirienne. Cette symbolique passe par l’incarnation de la fonction policière à travers la figure d'un représentant de l'ordre, agissant dans le cadre d'une institution policière. Cette analyse se propose de dégager les traits essentiels de la représentation filmique du policier en Côte d’Ivoire.La production filmique ivoirienne dans la représentation de la police ou du policier n'est pas aussi prolifique comparativement que celle produite par les réalisateurs burkinabés. Il s’agit donc d'étudier l'image des représentants de l'ordre mise en scène dans le cinéma, plus particulièrement dans les téléfilms. Cette représentation conduit à plusieurs interrogations : Quelles sont les pratiques policières mises en évidence dans les téléfilms ? Quels sont les phénomènes policiers que les réalisateurs tentent de mettre en image ? La 
mise en représentation filmique du policier n’est-elle pas un miroir des changements sociaux?

\section{Méthodologie}

\section{Echantillon}

Notre échantillonnage a fait le choix d'un certain nombre de téléfilms dans lesquels le rôle attribué au policier apparait. L'étude ne se limite pas à un seul téléfilm, mais à une diversité d'œuvres filmiques. Il s'agit de six téléfilms : Broutage.com 1, du réalisateur Alain Guikou, diffusé en 2013 ; Le Villageois 1 \& 2 (Les Gaous d’Abidjan), du scénariste Fidèle Kofi \& du réalisateur Hervé E. Lengani, produit en Mai 2009 et Les Aventures du Sergent Digbeu 1, 2 et 3, scénario et dialogue d’Alex Quassi, diffusé en Août 2005.

\section{Instrument de recueil des données}

L'étude a eu recours à l'observation documentaire ou étude de documents. Celle-ci porte sur des objets dont l'observation est indirecte grâce aux traces qu'ils ont laissées (N’Da, 2015). Nous avons observé ces documents filmiques desquels nous avons extrait la représentation du policier au travers du rôle joué dans les téléfilms.L’observation a consisté à voir avec précision ce qui se produit et à le noter pour pouvoir en faire une description, une analyse et, éventuellement, une critique. Un téléfilm ou un film donne une possibilité de visionnage illimité. On a visionné chaque téléfilm retenu plusieurs fois (au moins cinq fois) : visionnages répétés en vue de retenir, d'extraire et de noter les dialogues, les paroles des acteurs. Toutefois, l’on ne prétend pas avoir épuisé toutes les possibilités de lecture, il s’est agi avant tout de s’intéresser à la matière policière.

\section{Analyse des données}

Le traitement des données documentaire a porté sur l'analyse de contenu. Celle-ci est un ensemble de techniques d'analyse des communications. Elle s’applique à plusieurs champs : lettres, journaux intimes, tests projectifs, entretiens, livres, mass médias, réponses à des questions, etc. (Bardin, 1980). Il s’agit, ici, d’analyser des téléfilms, non pas avec un regard de téléspectateurs ou du cinéphile, mais d’un point de vue scientifique afin de mettre à jour la représentation filmique du policier. Dans cette étude, le rôle joué et attribué au policier dans les téléfilms a permis d’appréhender les types de représentation de celui-ci. 


\section{Resultats}

\section{Policier racketteur}

Les policiers ivoiriens ne bénéficient pas d'une bonne représentation dans les téléfilms. Ils sont souventdécrits comme de véritables racketteurs embusqués aux bords des routes. Leurs proies, les plus habituelles, sont les chauffeurs de wôrô-wôrô, de taxis et de gbaka.

Dans le téléfilm “Le villageois”' d’Hervé Lengani (2009), à un barrage de fortune, le sergent de police Kahizer arrête le taxi emprunté par Tapé Gaston (le villageois) : "Police nationale, contrôle de routine! Permis de conduire et les pièces afférentes au véhicule, s’il vous plaît!" Après vérification, le chauffeur a toutes les pièces requises. L'agent de police s'adresse à son collègue : "ça sent mauvais, les pièces sont au complet; tu vas créer ? "; Sergent Pokou : "ça c'est un petit problème, on va voir qui est qui ici. Montre-nous ton triangle de signalisation, arrière et avant, extincteur...». Le chauffeur a tout présenté ; le sergent de police Pokou invente alors une infraction : " article 320a, entrave à la liberté animale...ici on n'attache pas les pattes d'un animal...attaque verbale...ce qui correspond à 5000fCFA». Ce sont des fautes imaginaires pour soutirer de l'argent aux citoyens. Ainsi, pour la présence d'un signe distinctif d'appartenance à une religion (un chapelet) dans le téléfilm "Les Aventures du Sergent Digbeu", le policier dit au chauffeur : «Toi, tu es dangereux, tu ne sais pas que c'est interdit... Je ne discute pas avec vous. Mon frère, si tu veux me donner les dix milles tu me donnes, si tu ne veux pas tu laisses, la voiture va aller en fourrière... ».

Le devoir du policier est de respecter et de faire respecterles libertés fondamentales du citoyen, à savoir la liberté de circuler, de réunion, de croyance et d'opinion conformément aux articles 18,19 \& 20 de la Déclaration des droits de l'homme de 1948. Le policier n'a pas le droit d'empêcher les individus de circuler librement ou d'abuser de son autorité, de son statut, à l'égard d'honnêtes citoyens qui subissent ses tracasseries. L'expression «tracasseries policières » doit se comprendre comme des ennuis que subissent les automobilistes du fait de l'action des forces de l'ordre chargées des contrôles routiers. En réalité, il s'agit d'un maquillage linguistique tendant à donner un aspect plus présentable au racket orchestré par des agents investis de l'autorité publique (Yébouet, 2004). Le racket policier constitue une entrave à la libre circulation des personnes et des biens.

Des barrages de fortune, des contrôles inopinés sont mis en œuvre dans le seul but de soutirer de l'argent aux citoyens qui, malheureusement, se trouvent sur leur chemin. Au cours des contrôles sur les routes, les automobilistes arrêtés, pour éviter d'être conduit au poste de police, sont obligés de laisser quelques pièces d'argent ; même s'ils sont en règle et n'ont 
commis aucune infraction relative au code de la route. Dans "Le Villageois", après plusieurs heures passées à un barrage de fortune, les policiers, projetant de soutirer de l'argent aux chauffeurs, ne voient pas de véhicules circuler. Fatigués d'attendre, ils se posent un certain nombre de questions sur cette dure journéeoù il n'y a personne à racketter. C'est ainsi que l'agent de police Kahiseur dit à son collègue : «aujourd'hui ce n'est pas bon...je ne comprends pas ». Au sergent Pokou de répondre : "c'est quelle journée, personne ne passe.»Puis, ces policiers voient venir un piéton. Ils s'empressent alors vers ce dernier et l'interpellent: "hé viens-là,... tes pièces... », lui demande le sergent Pokou. Le piéton répond les avoir laissées à la maison. N'ayant pas sa pièce d'identité, l'agent lui demande en retour de l'argent sinon, il ne pourra partir : "fais quelque chose...si tu ne donnes rien tu vas "krô"' (dormir) avec nous...tu n'as rien et tu te promènes, quand on n'a rien, on reste à la maison ». L’interpellé, pour éviter de perdre du temps à ce barrage, leur remet une pièce de monnaie pour étancher leur soif : «Voilà “deux gros” (50fCFA) vous allez boire de l'eau ».

Le racket policier est aussi représentée par le scénariste Alex Quassi (2005) dans le téléfilm “'Les Aventures du Sergent Digbeu”. Le sergent Digbeu se rend sur la route. Il arrête plusieurs véhicules. Le premier véhicule s'arrête, le chauffeur descend et lui tend les pièces. Le sergent : "Moi je t'ai dit que...» Le chauffeur : "ça hé dedans ».Le sergent : "Il faut enlever, tu vas me donner, tu penses que ceux qui sont dedans (véhicule) ne savent pas ? » Pour le chauffeur, seul le billet de banque suffit, aucune vérification de documents afférents au véhicule n’est nécessaire. Le sergent pose cependantla question au chauffeur qui lui tend les pièces. En retour, le chauffeur, connaissant la combine, répond qu'il a placél'argent entre les pièces qu'il tient dans la main. Le policier répond qu’il n’y a rien à cacher car personne n'ignore cette pratique. Le chauffeur lui donne un billet de mille francs. Puis, on passe au second véhicule. Le sergent : " hé mon petit, c'est toi !»Le chauffeur dit: «aujourd'hui là c'est mou dèh! », ce qui signifie que la journée est difficileet que les clients se font rares. Pour le policier, peu importe que la journée soit bonne ou pas; il attend que le chauffeur de taxi lui donne de l'argent car, en fait, il n'est pas présent sur la route pour contrôler les pièces : "Là il faut faire un effort, tu vas donner. Je vais faire quoi avec pièces ! » Après avoir reçu un billet de mille francs, le policier congratule le chauffeur: "Tu es un petit qui est courageux». Ensuite, troisième véhicule. Le sergent : "Ah ! Viens ici ; tu as réglé quoi !» Le chauffeur : "j’ai réglé la journée !». Le sergent tient une feuille en main sur laquelle se trouve l’immatriculation des véhicules rançonnés durant la journée. Après vérification, le policier laisse le chauffeur partir car il a déjà régléla somme qu’il faut pour la journée. 
Les téléfilms montrent que le phénomène du racket est souvent ordonné par le supérieur hiérarchique du policier. Dans “'Le Villageois”, Tapé G. est conduit au poste de police... Le commissaire demande au sergent d'offrir à boire à ce dernier. N'ayant pas d'argent pour acheter à boire, le sergent de police s'adresse alors au commissaire : «vous ne m'avez pas remis de l'argent ! " Le commissaire : "C'est à moi tu dis ça ! Je vous ai appris à survivre sur le terrain, trouve toi un taxi... ». Le mot est lâché. Le commissaire intime l'ordre aux policiers d'aller soutirer de l'argent aux chauffeurs de taxi. Le commissaire de police est, en effet, souvent l'initiateur et l'organisateur des rackets sur les routes. Le racket est une organisation criminelle se présentant sous une forme pyramidale que les réalisateurs tentent de mettre en évidence.

Par exemple, dans le téléfilm “'Les Aventures du Sergent Digbeu’, le sergent arrête un taxi et tend le bras à l'intérieur : «Fais vite, la douane !» Le chauffeur lui tend les pièces du véhicule. "C'est quoi ça, je fais quoi avec papier, donne-moi les vrais papiers !», dit le sergent. Le passager dans le véhicule, qui est en fait un commissaire de policeintervientet demande au sergent embusqué de lui remettre de l'argent : "bonjour jeune et le mouvement ? " Le sergent se met au garde-à-vous : "C'est dur, on fait un effort mais ça ne va pas du tout». Le commissaire : " file-moi 5tickets (donnes-moi 5000!) Le sergent : «5000fCFA matin-là ? " "Ne déconne pas », dit le commissaire. Le sergent lui remet les 5000fCFA. Ensuite, il fait remarquer que les commissaires, en général, sont des racketteurs et celui-là, en particulier, ne donnera pas au chauffeur, la somme due au kilométrage parcouru : "Ils aiment trop racketter, les commissaires ; ce taxi là même, il ne va pas payer ».

Après le terrain, le sergent retourne au poste de police pour faire le bilan de la journée, en faisant croire à son supérieur que la journée a étédifficile pourpouvoir conserver un peu d'argent... Le sergent Digbeu : «ça n’a pas été facile; les gens, on dirait qu'ils ont changé d'itinéraire ». Le sergent tend des billets de banque à son chef hiérarchique. "C'est tout ce que tu as eu? C'est sûr que si je passe à la fouille, je ne trouverai plus rien !, s’interroge le commissaire. Le sergent: "J'ai dû oublier lorsque je faisais la monnaie...voilà ». Le commissaire : "C'est tout...si tu continues de m'amener des miettes, je pense que je vais te fixer un montant maintenant». "Chef, je vais travailler! Je vais doubler! Je double les taxes !», répond le sergent. «Il faut que ça carbure, Tu envoies des miettes, ce n'est pas bon », lui répond le commissaire.

Cette organisation criminelle s’observe également dans le téléfilm “Le Villageois” dans lequel le commissaire s’adresse au sergent Kahiseur en ces termes : «Et la journée d'aujourd'hui !» Le sergent répond : «très bien mon commissaire ». Il dépose des pièces d'argent et des billets de 
banque sur la table. Le commissaire, très heureux du racket, veut, non seulement assurerla promotion du sergent, mais également souhaite l'affecter dans une zone où le trafic routier est plus dense afin qu'il soutire encore plus d'argent : «tu travailles bien...ce qu'on peut chercher à faire, c'est de t'augmenter un «V» encore. Mieux, je vais t'envoyer sur la route d'Azaguié...si tu vas là-bas, tu travailles pour moi. Il était sur une route difficile et il a fait ça! ». C'est au tour du sergent Pokou de rendre compte à son chef hiérarchique. "Fais-moi le point de la journée, pas de commentaire, tu me vides tes poches. C'est quoi ça ? C'est la recette du jour ça ? » dit le commissaire. Mécontent, le commissaire fait appeler le sergent Kahiseur et lui donne cet ordre en parlant du sergent Pokou: «tu vas me vider les poches de ce monsieur ». Le sergent Kahiseur s’exécute. Plusieurs billets de banque sont découverts, y compris des téléphones portables et des bijoux. Tout ceci avait été dissimulé dans les différentes poches de la tenue du sergent Pokou. «Si tu veux être bijoutier, tu me dis ; si tu veux vendre des portables au black, tu me dis !» s'exclame le commissaire. En fait, le sergent de police prend les objets tels que des bijoux ou des téléphones comme des gages pour faire pression sur les chauffeurs qui n’ont pas versé la somme attendu. En effet, lors des contrôles au barrage, le policier exige une somme à verser. Si les chauffeurs ne l'ont pas, ils déposent en contrepartie des objets dontla valeur est plus élevée que la somme à verser pour que les chauffeurs reviennent après les chercher en payant la somme : «Quand les chauffeurs n'ont pas de liquide, ils mettent ça en garantie et le lendemain ils viennent prendre au barrage ; ils donnent l'argent pour récupérer...les passagers qui n'ont pas d'argent, ils donnent ça en lieu et place ». Les agents sur le terrain rackettent donc pour le commissaire. Après la moisson, le commissaire fait, lui-même, le bilan de la journée. Constatant que ses agents ne versent pas la totalité des gains attendus, le commissaire décide de les muter au poste de police : «tu n'iras plus sur la route, tu vas rester, ici, au comptoir ». Aussi, pour éviter de se faire gruger par ses agents, le commissaire projette se rendre lui-même sur le terrain. Pour lui, il y a un minimum à verser dans la journée ; en deçà, il considère que ces agents ne reversent pas la totalité du montant attendu : «Il doit verser 125000fCFA. La dernière fois, il a versé 67000, la différence qui va donner ? Quand moi je suis sorti de l'école de police, on m'a donné un sifflet, j'ai ça ici ; moi-même je vais aller sur la route..."

En somme, les policiers opèrent soit en groupe - dans ce cas le chef de poste reste dans la guérite tandis que les subalternes interpellent et lui envoient les uns après les autres les usagers de la route pour lui verser les pourboires - soit individuellement sur le terrain, mais avec l'approbation du supérieur hiérarchique, en l’occurrence le commissaire de police. Dans ce cas, le chauffeur interpellé par un coup de sifflet, connaissant la « combine » 
glissera, entre les pièces du véhicule, la somme “conventionnelle”, véritable certificat de dispense de contrôle. Le racket est une pratique mise en œuvre au vu et au su de tous puisque cela s'opère au niveau des barrages routiers et à chaque carrefour. Ils persécutent les citoyens et les obligent à leur verser de l'argent. Le policier est chargé de protéger et de rechercher les délinquants qui s'attaquent aux biens des citoyens, toutefois, certains policiers, par leur pratique, peuvent être assimilés à des ripoux.

\section{Policier ripou}

Si le racket policier est une forme d'extorsion de fonds, la corruption, " c'est le fait d'accepter ou de réclamer une rétribution en échange d'une violation des devoirs professionnels » Dieu \& Dupont (2001). Il s'agit, alors de fermer les yeux sur des infractions réelles et d'être ainsi complice des délinquants.

Dans “'Les Aventures du Sergent Digbeu”, Mahoud est un vendeur de pneus qui fait de l'import/export. Pour éviter de payer la totalité des frais de douane, il a remisune somme d'argent au douanier pour que sa marchandise puisse sortir du port. Le douanier partage cette information avec le commissaire de police afin que celui-ci puisse avoir sa part. Informéde la fraude, le commissaire demande au sergent de rendre visite à Mahoud en vue de récupérer le somme due. Pour y arriver, le commissaire demande au sergent d'usurper le titre de lieutenant: «tu connais mon ami Daplé, l'inspecteur de douane, il m'a fourni un bon tuyau à propos de Mahoud, le vieux Libanais, vendeur de pneus. Il a importé la semaine dernière pour près de 100 millions de francs de marchandise avec l'aide de Daplé et tient toi bien, il n'a payé que 5 millions pour le dédouanement au lieu de 17 millions. $\mathrm{Tu}$ vas descendre sur le terrain et récupérer les 3 millions... tu as ma permission de jouer le rôle de lieutenant.». De retour de la mission à lui confiée, le sergent rend compte au commissaire: "ça c’est pour Mahoud».Le commissaire Blé répond: "J'espère que le compte y est...».Dans “Le Villageois”, pour étouffer un procès-verbal, en échanged'une contrepartie, le commissaire s'adresse au parent d'un suspect, joint au téléphone, en l'invitant à venir remettre ce qu'il faut le plus rapidement possible pendant qu'il peut encore mettre fin à la procédure : « il faut vite faire pendant que le problème est à mon niveau ; une fois que c'est au parquet, je ne peux plus intervenir. Il sera déféré demain...tu sais, le chauffeur de la MACA (Maison d'Arrêt et de Correction d'Abidjan) est très rapide...»

Cette représentation du policier ripou s'observe aussi dans le téléfilm “Brouteurs.com” du réalisateur Alain Guikou (2013). Faut-il rappeler que le 'broutage”' est un mécanisme mis en place par des délinquants pour soutirer 
de l'argent à des victimes via internet (terminologie renvoyant à l'adage selon lequel ''le mouton broute là où il est attaché')'. En d'autres termes, dans les transactions commerciales, bancaires et financières se faisant sur la toile et à travers le monde cybernétique les cybercriminels trouvent des opportunités criminelles multiples, leur permettant de ''brouter' (Akadjé, 2011). Dans «Brouteurs.com », Géraud \& Camso sont deux brouteurs qui exercent leur activité criminelle avec la complicité de deux policiers ripoux : Naja \& Blanco. Ces flics suivent les faits et gestes des brouteurs dans les différents espaces fréquentés par ceux-ci : les bars, les salles de jeux et leur domicile. Leur seul but pour ces policiers est de tirer profit des manœuvres frauduleuses des brouteurs, non de les réprimer.

Ainsi, les policiers font irruption dans la salle de jeu où se trouventdes brouteurs. Blanco prend l'argent déposé sur la table de billard par les brouteurs et réclame encore plus, en poussant une petite assiette devant Géraud pour que celui-ci y mette de l'argent: "la mise!» Géraud met des billets de banque et pousse l'assiette vers Naja. Naja à son tour repousse l'assiette car les billets sont insuffisants. Géraud ajoute à nouveau des billets. Satisfait du montant reçu, Blanco fait un geste des doigts en pistolet en relevant, non seulement les brouteurs travaillent pour eux mieux qu'ils sont de la police criminelle: "vous savez, vous les enfants, vous êtes nos café-cacao et nous nous sommes de la police criminelle ».La recherche du gain facile anime donc non seulement les brouteurs mais également certains policiers. La corruption est une gangrène pour la société ivoirienne et celle-ci a du mal à trier le bon grain de l'ivraie. Une telle collusion ne peut qu'encourager les délinquants à multiplier leur activité. A l'arrivée du brouteur (Géraud) et de sa copine, dans le bar, le policier "chante”' les louanges de son partenaire. Quelques heures plus tard, Géraud s'apprête à quitter le bar. Le policier ayant les yeux fixés sur le brouteur l'interpelle: " Géraud, tu m'oublies ou quoi ?...il y a la boisson et après, on doit aller à l'hôtel...» Géraud lui remet alors des billets de banque. "Le compte n'est pas bon!» s'exclame le policier. Et, il se met en colère : «Donne-moi le fric ! Donne-moi le fric !» Après que Géraud ait ajouté des billets, le policier tout heureux dit : «Ah ! Ça c'est bon ! »

Enfin, ces policiers ripoux font des rondes spécifiquement autour du domicile de Géraud. Plus grave, ils y interviennent chaque fois qu'ils ont besoin d'argent. Blanco : " excusez-nous d'avoir interrompu votre séance de travail. On a un souci. Je suis en mission avec mes potes; tu sais on roule, on roule...tout à coup une panne sèche ; on fait quoi dans ma situation ?»Le brouteur Géraud ayant compris les motifs pour lesquels ces deux ripoux sont présents à son domicile, leur pose la question suivante : «vieux père, vous voulez combien ? » Blanco : «juste cent mille FCFA». Géraud : "on n'a pas cent mille, les poches sont vides. " Blanco: «c'est qu’à même toi, 
Géraud. Et lui aussi il n’a rien ? Gagnons en temps. " Après avoir reçu les billets, le policier Blanco vérifie la somme : «La confiance n'exclut pas le contrôle...Naja, le compte y est». Naja: "encore une fois, toutes nos excuses pour le désagrément. "

Certes, les brouteurs sont harcelés par des policiers violents et corrompus mais, en retour, pour ceux-ci la complicité des flics ripoux leur garantit l’impunité. Ainsi, ils sollicitent la collaboration des policiers lorsqu'ils préparent une escroquerie qui compte leur rapporter beaucoup d'argent afin que chacun ait sa part du gâteau. Ce sont ces phénomènes policiers que les réalisateurs mettent en image ; une situation qui n'est pas loin de la réalité (Akadjé, 2011).Toujours, dans le téléfilm “Brouteurs.com”, Camso, le brouteur, dit à Géraud : "il ne faut pas oublier les deux flics ripoux, parce qu'on aura besoin d'eux quand l'arabe va arriver ici. Après le retrait du butin, il faut que tout le monde ait sa part de gâteau.» Géraud appelle alors le policier Blanco pour qu’il vienne récupérer sa part du butin : "vieux père s'était pour voir si vous pouvez passer.» Les policiers ripoux arrivent au domicile du brouteur. Les brouteurs remettent aux policiers une part de leur butin. Puis, ils leur font part d'une “affaire' et ont besoin de l'appui de policiers. Il s'agit pour les policiers d'escorter la voiture à l'arrivée du saoudien (la victime des brouteurs), c'est-à-dire accompagnerpour guider, surveiller leur victime pendant le trajet jusqu’à sa résidence. Telle est l'essentielle de cette “affaire”'. En retour, les brouteurs proposent aux policiers une forte somme d'argent : " Dimanche prochain, on a une fête ici, on aimerait bien vous inviter. On a prévu un petit cadeau pour vous. 500 mille franc pour le carburant; vous n'êtes pas au bout de votre surprise. On a une affaire pour vous ». Géraud : " une toute petite affaire qui va vous rapporter 2 millions. On a un client qui arrive la semaine prochaine et on aimerait que vous l'escortiez. En retour, vous aurez 2 bâtons. » Les policiers ripoux entrent en négociation en vue de revoir le montant proposé à la hausse : "Nous pour une telle escorte, c'est petit, trop petit. 5 bâtons (cinq millions), c'est non négociable, qui ne dit rien consent. » Le brouteur (Géraud) accepte la proposition des policiers ripoux, c'est-à-direcinq millions: "Ne vous en faites pas, tout va bien se passer et à la fin vous aurez vos cinq millions.»

En plus de l'exploitation des brouteurs, les flics ripoux collaborent avec des receleurs avec lesquels ils font des affaires. Ces délinquants ont mission de vendre les objets que proposent les policiers. Ainsi, on voit des policiers ripoux allant à la rencontre d'un receleur afin de partager un certain nombre d'informations sur les brouteurs dans le quartier. En retour, il ne sera pas arrêté pour l'acte de recel. Blanco s’adresse à John Poloss : "voici le deal, on te laisse faire tes petites activités de recels et toi tu nous donnes toutes les informations sur les gros coups. John Poloss refuse cette 
proposition ; il préfère le suicide ou la prison. Pour lui, la seule condition pour qu'il dénonce ses camarades, c'est qu'il soit l'unique receleur des objets de valeurs saisis illégalementpar ces policiers : "Donc vous me laissé bosser et en retour je vous vends mes amis. C'est ce que vous me proposez ...vous êtes de la P.J, non! Je préfère que vous m'envoyer en prison. Je marche avec vous à une seule condition : c'est à moi que vous devrez maintenant revendre tous les objets de valeurs que vous bloquez quand vous montez sur le terrain. » Le policier Blanco accepte la proposition de John Poloss: "il veut être notre receleur. Le deal me va; toi notre receleur, Ok ». John Poloss: « ça roule, donc je suis dans mon coin, j’attends vos objets volés.»

Quelque temps après, les policiers interpellent John Poloss et le conduisent dans un bar en vue d'avoir quelques informations, suite à l'accord conclu entre eux. Le receleur ne comprend pas l'indiscrétion des policiers ; car il peut être reconnu dans l'entourage comme une personne travaillant avec les policiers : "mes vieux, vous n'avez pas trouvé un endroit plus discret? Tel que nous sommes exposés les gens sauront que je suis votre mouchard.» Blanco : "Tu as quoi pour nous ?» John Poloss répond : «ah! Mon vieux c'est à toi de me dire ce que tu as avant que je chante comme Bailly Spinto (nom d'un artiste-chanteur), c'est ce qui est la vérité. » Car, au terme du contrat, les policiers devraient lui vendre les objets saisis avant de recevoir toute information. Le policier (Naja) lui tend une montre. John Poloss réagit sur la valeur de la montre : «Vieux, ce n'est pas une originale ; sur le marché, je n'aurai rien c'est du chinois. » Naja : "Il n'y a rien d'original de nos jours. » Puis, John Poloss donne des informations qu'il a en sa possession aux policiers en vue d'avoir davantage d'argent : "ce sont de vraies informations qui vont vous faire gagner de l'argent. Nous, c'est vous et moi, nous sommes maintenant associés. Ne doutes pas de mes informations ; les noms et adresses nous ferons gagner des millions. » Naja : "nous sommes des policiers, on ne joue pas avec nous. " John Poloss : «Mes vieux frères, si on veut avoir plus d'argent, il nous faut évoluer dans notre stratégie de vol...»

Ces policiers ripoux sont des partenaires, des collaborateurs, ou plutôt des complices des délinquants. Ces délinquants prennent le risque de continuer leur activité délictueuse car, ils savent qu'ils ne seront ni arrêtés ni poursuivis pour leurs manœuvres frauduleuses par les policiers. Au contraire, ils partagent une partie des sommes d'argent obtenues par l'escroquerie via Internet et autresmoyens, pour s'assurer les complicités nécessaires.

\section{Policier violent}

La violence a toujours accompagné la vie des sociétés : à l’origine étroitement liée à la survie du groupe humain, la violence s'est perpétuée 
avec la complexification croissante des sociétés ; elle s’est maintenue malgré les progrès de la vie sociale. La violence est un concept complexe à définir bien qu'elle semble aisée à reconnaître dans la vie de tous les jours (Richoud, 1993). La violence est un emploi brutal de la force en vue de tuer, blesser, intimider ou contraindre quelqu'un contre sa volonté. L'étymologie fait remonter le terme au mot latin violentia: “ abus de la force " (Pérouse, 1997). Elle peut être définie comme une atteinte portée à l'intégrité physique ou à l'intégrité morale dans une situation d'interaction entre un ou plusieurs acteurs (Michaud, 1992).

La difficulté que l'on rencontre à établir un consensus autour d'une définition de la violence tient au fait que le concept de violence est variable dans le temps et dans l'espace. En effet, on ne se représente pas la violence de la même façon selon que l'on appartient à une civilisation plutôt qu'à une autre, à une époque qu'à une autre. La violence d'aujourd'hui ne sera pas peut être celle de demain.

Des différentes catégories de violence qui ont inspiré toute une littérature. Ce sont les violences policières qui constituent l'essentiel de notre étude. Il s'agit de l'usage abusif de la force ; c'est-à-dire un comportement déviant des forces de police. La typologie que dresse François Dieu (1995), à propos de la violence policière peut permettre de mieux analyser l'attitude des forces de police en Côte d'Ivoire telle qu'elle est représentée par les téléfilms. Selon François Dieu le passage du singulier au pluriel illustre l'hétérogénéité concrète des phénomènes que l'on regroupe, souvent de manière sommaire, sous le concept simplificateur de "violence policière". La rudesse, l'agressivité sont parfois considérées comme des attitudes naturelles de la police, étant donné qu'elle peut faire usage de la force, cellequi résulte de l'utilisation normalisée et maitriséede la violence dans une tâche policière autorisée par une autorité légitime (Akadjé, 2003). Apparemment, la police elle-même se voit de cette façon : c'est ainsi que nombre de policiers estiment que seule la rudesse leur permet de réussir. Le citoyen n'ignore pas cette rudesse.Mais Il exige une action rigoureuse contre les délinquants, les criminels, mais pas contre lui. D’où l’idée que la police est autorisée à faire ce qui correspond au vœu refoulé de tout un chacun : employer la violence contre les autres (Steinhilper, 1979). Lorsque cette agressivité s'exerce contre la population, celle-ci reproche à la police de bafouer ses droits; de ne pas respecter les libertés fondamentales. Aussi se plaint-elle de ce que, pour résoudre un conflit, la police ne recherche ni ne favorise la communication avec son "adversaire", mais applique principalement des stratégies de puissance, d'agressivité et de violence. Il s'agit, ici, de l'usage abusif de la force, plus précisément de la menace instrumentale de la brutalité que les réalisateurs mettent en image dans les téléfilms. 
Dans “'Le Villageois”, Tapé G. en provenance de son village, un coq à la main, n'ayant pas sa pièce d'identité, les agents de police le font descendre par la force du taxi et demande au chauffeur de partir. Il est brutalisé, mis au sol et conduit manu militari au commissariat. Arrivé au poste de police, il dit aux agents que son frère est Bahi Roger, le Directeur de la Police Nationale. En vue de vérifier cette information, celui-ci est joint au téléphone par le commissaire Digbeu-Cravate. Après avoir échangé avec Bahi Roger, qui confirme les dires de Tapé G., le commissaire change de langage à l'égard de ce dernier. Le commissaire reproche alors aux agents de ne pas réserver un bon accueil et de ne pas bien traiter le citoyen : "C'est comme ça on traite un citoyen, s'il n'a pas de pièce c'est à vous de lui dire ce qu'il faut faire; vous venez avec un monsieur au commissariat, faites le asseoir, donnez-lui à boire...Amenez une bière !» L'attitude du commissaire Digbeu-cravatte montre qu'avoir un policierparmi ces connaissances, surtout si celui-ci est le Directeur de la police, ça peut toujours servir. Dans le cas contraire, le citoyen lambda subira au poste de police les brimades et la brutalité policière. Par exemple, dans “Brouteurs.com”, le policier, après une interpellation, met le brouteur manu militari dans le coffre de la voiture sans lui avoir lu ses droits.

La police étant faite pour les personnes et non contre elles, comme certains paraissent le penser, elle ne peut agir sans bénéficier de l'acceptation de ses méthodes par la population. A défaut d'une acceptation suffisante de ses méthodes, la police est vue comme une institution opposée au public, situation particulièrement néfaste pour faire face à la délinquance. Le policier érige, par exemple, les « interrogatoires » très musclés en pratique habituelle de recherche de la vérité. Pourtant, le policier n’a pas le droit de faire avouer sous la contrainte un acte à un prévenu. Or, dans "Un Crime à Abidjan', le suspect est brutalisé, torturé et reçoit des coups du policier qui veut obtenir des aveux. La cruauté de la police envers le suspect et l'intimidation par la menace qu'elle exerce sur les citoyens pour obtenir des aveux ne peuvent qu'en donner une représentation négative dans les téléfilms.

L'intimidation, la menace sont donc présentes dans les rapports entre policier et citoyens. Elles sont mises en scène par les réalisateurs en vue de montrer la brutalité du policier ivoirien. Dans les téléfilms ivoiriens, elles sont présentées comme la norme, comme le moyen normal pour obtenir l'obéissance à une requête. Dans, Broutage.com, par exemple, les brouteurs, Géraud et Camso, font un retrait d'un montant de 3.200.000Fcfa. Les agents de police se rendent au domicile de Géraud et le menacent en réclamant de l'argent. Le policier Blanco : «où allez-vous ce matin ? C'est bizarre, ce matin j'ai pensé à vous! Surtout toi Géraud tu m'as manqué... Fais-moi un câlin! C'est mon champion préféré ! Donnes pour moi !» Le brouteur fait 
semblant de ne pas comprendre la demande du policier : «je ne sais pas de quoi tu parles ». Le policier Naja, mécontent de la réponse du brouteur, menace et réagit avec violence en faisant des gestes; le policier fait un emploi brutal de la force par la menace en vue d'intimider ou de contraindre le “brouteur"' contre sa volonté : "tu ne comprends pas, lorsque je vais foutre mon flingue dans ta sale gueule de petit brouteur de mède...» Blanco propose alors à Géraud de se retirer pour en discuter : «ce ne se serait pas mieux qu'on aille discuter ailleurs, à l'intérieur entre grand frère et petit frère... Voyant une bouteille de champagne et des verres, il ajoute« Géraud, tu as fait la fête ici et là tu commences à oublier ton grand frère, ce n'est pas bien.» Le policier menace le brouteur : "Mon fric ou bien dis-moi, tu as... » Géraud remet l'argent à Blanco. Blanco : «Le compte n'y est pas ! Le compte me dit qu'il n'y est pas! Le compte n'est pas, ne joue pas au con avec moi! Pour la prochaine fois, tu fais un faux coup, tu ne m'oublies pas ? » Le policier procède alors à une fouille corporelle et retire quelques billets des poches de Géraud.

Toujours dans cette série, le policier Naja interpelle le receleur John Poloss. Pas content de la réponse de ce dernier, le policier s’énerve, descend du véhicule, donne des coups à John et le met dans le véhicule...Le policier (Naja) : "Moi j’ai une proposition plus alléchante à te faire. Je mets une balle dans ta tête et je pose mon arme sur ton corps en pointe de légitime défense ». Cette représentation est une menace instrumentale de la brutalité du policier en vue d’intimider l’interpelé. Le comportement déviant du policier le contraint à céder à la requête de ce dernier. En effet, la peur d'être abattu sous prétexte delégitime défense par le policier l'oblige à accepter la requête du policier. John Poloss : «Ok! Je marche avec vous. » Dans "Le Villageois", le commissaire s'adresse au sergent: "je ne sais pas quel concours tu as passé pour être policier. On est obligé de tout faire dans ce pays...petit (chauffeur de taxi) donnes les pièces, il a cotisé ?» Le sergent: « il a ses pièces au complet !» Le commissaire : "on est au soleil...petit (chauffeur) tu as cotisé...je vais te gifler, tu me connais ; tu vas faire, tu vas descendre tout à l'heure. Regarde-lui là, tu te prends pour qui, tu la boucle! » En brutalisant le chauffeur, il ouvre la portière, demande au passager arrière de se taire. Le comportement du policier est inquiétant. L'usage de l'arme de service pour menacer et intimider le citoyen, la brutalité sont les actes de déviance que les réalisateurs représentent souventdans les téléfilms. En un mot, la brutalité, l'usage abusif de la force et l'intimidation apparaissent comme des moyens pas «illégaux » mis en œuvre par la police dans les téléfilms. Or, la violence déviante est celle qui s’inscrit en dehors du cadre légal et légitime de l'action policière, quels qu'en soient les buts ou les moyens mis en œuvre. 
Il est vrai que le travail policier montre une diversité des tâches assurées sur le terrain dans la pratique quotidienne ; une réalité aux aspects extrêmement différenciés avec des pratiques très diverses. Toutefois, les policiers ont été formés pour assister les citoyens, pour assurer leur sécurité et, surtout les aider à s'épanouir librement dans le pays. Lorsqu'on constate le contraire, il y a lieu de s'inquiéter. Cette inquiétude est l'emploi brutal de la force en vue d'intimider ou de contraindre les citoyens contre leur volonté.Les citoyens et les réalisateurs, en interpelant le corps social et l'autorité en charge de la sécurité, souhaitent contribuer à un changement de mentalité pour que règne la sécurité.

\section{Conclusion}

Les années 1970 furent marquées, en Côte d’Ivoire, par des comportements policiers qui semblaient plutôt vertueux : intégrité morale, refus de toute compromission, application stricte de la loi, même à l'encontre de parents. Même s'il y avait des policiers qui exigeaient de l'argent des propriétaires de taxi et de minibus, dans l'ensemble, l'image que les gens se faisaient du policier apparaissait comme assez positive (Brillon, 1980). Les forces de l'ordre semblaient inspirer plutôt le respect, la confiance. La seule présence d'un gendarme ou d'un policier en un lieu était un gage de sécurité. Les policiers du rang semblaient ne pas pratiquer le racket ou ne pas céder à la corruption. Ils faisaient honneur à leur corporation et conféraient à la profession l'image d'une autorité exemplaire. Mais comme une seule mauvaise graine peut ternir cette image, se sont multipliés les policiers racketteurs, corrompus, escrocs et voleurs. Le racket policier porte préjudice à l'économie ivoirienne vu les sommes d'argent que prennent certains policiers aux citoyens chaque jour. Une étude menée par la Banque mondiale a, par ailleurs, révélé que l'Etat ivoirien perd en moyenne 350 milliards de FCFA (environ 500 millions d'euros) par an à cause du racket (Xinhua, 2014). C’est ce que les réalisateurs tentent de dénoncer, dans les téléfilms. Policiers et délinquants sont, parfois, difficilement discernables l'un de l'autre.Le racket, la corruption et l'intimidation par la menace de la violence sont les phénomènes policiers que les réalisateurs mettent en image dans les téléfilms Ivoiriens. Une représentation qui n'est pas loin de la réalité. Aujourd'hui, l'on semble arrivé à un stade où celui qui fait correctement et honnêtement son travail, et se contente de son seul salaire est considéré comme un imbécile ou un extra-terrestre. Ces comportements déviants traduisent le malaise de la société ivoirienne en générale, surtout en ce qui concerne la corruption, les pots-de-vin pour tout service dans l'administration. Tous les discours, colloques, séminaires et forums sur la bonne gouvernance ne serviront absolument à rien si des actes clairs ne sont pas posés, si les vrais coupables ne sont pas punis et, surtout, si l'on 
n’inculque pas une nouvelle mentalité aux jeunes. On ne peut atteindre les objectifs fixés qu’avec l’adhésion et la moralisation du peuple et du policier.

\section{References:}

Akadjé, M. (2003). Sécurité publique et sécurité privée en Côte d'Ivoire. Thèse. Université des Sciences Sociales de Toulouse 1, France, 410p.

Akadjé, M. (2011).Cybercriminalité et '‘broutage”' à Abidjan. Revue Internationale de Criminologie et de Police Technique et Scientifique, $\mathrm{n}^{\circ} 3$, Juillet-Septembre, pp.299-310.

Bardin, L. (1980). L'analyse de contenu. Paris : PUF Le psychologue.

Bittner, E. (1993). « De la faculté d'user de la force comme fondement du rôle de la police », les Cahiers de la Sécurité Intérieure, n³, IHESI. pp.224235.

Brillon, Y. (1980). Ethno Criminologie de L'Afrique Noire. Vrin. Paris : Presses universitaires de Montréal. 368p.

Chablon, M. \& Léonard, L. (2007). Insécurité, police de proximité et gouvernance locale. Paris : L’Harmattan. 175p.

Dieu, F. (1995). « Eléments pour une approche socio-politique de la violence policière ». Déviance et Société, vol.19, n¹, pp.35-49.

Dieu, F. \& Dupont, B. (2001). "L'évolution des connaissances et des politiques en Grande-Bretagne ». Les Cahiers de la Sécurité Intérieure, n44, IHESI, pp.9-36.

Loubet Del Bayle, J-L. (1992). La police, approche socio-politique. Paris : Montchrestien. 158p.

Loubet Del Bayle, J-L. (2001). «Vers une monopolisation policière du contrôle social ? » les Cahiers de la Sécurité Intérieure, n44.

Loubet Del Bayle, J-L. (2006). Police et politique. Paris : L’Harmattan. 318p.

Loubet Del Bayle, J-L. (2012). De la police et du contrôle social. Paris : Ed. Cerf. 220p.

Michaud, Y. (1992). La violence. Que sais-je ? n²251, Paris : PUF, 127p.

Monet, J-C. (1993). Police et Société en Europe. Paris : La Documentation française. 338p.

N’Da, P. (2015). Recherche et méthodologie en sciences sociales et humaines. Paris : l'Harmattan. 129p.

Philippe, O. (1999). La représentation de la police dans le cinéma français (1965-1992). Paris : L’Harmattan. 480p.

Pérouse de Montclos, M. A. (1997). Violence et sécurité urbaine en Afrique du Sud et au Nigeria, essai de privatisation: Durban, Johannesburg, Kano, Lagos et Port Harcourt. Paris : L'Harmattan, Collections logiques politique, Tome 1 \& 2, 303p. \& 480p. 
Richoud, V. (1993). Femme et violence dans la police. Mémoire DEA, UT1, 157p.

Rocher, G. (1968). Introduction à la sociologie générale. T.1: L’action sociale, HMH. 322p.

Steinhilper, G. (1979). «Violence et police », $3^{\mathrm{e}}$ Colloque Criminologique 1977, in La police et la prévention de la criminalité, Conseil de l'Europe, Affaires juridiques, Etudes relatives à la recherche Criminologique, Volume XVI, Strasbourg. pp.65-102.

Yébouet, H. (2004). « Le phénomène des tracasseries policière sur les routes en Côte d'Ivoire : le cas d'Abidjan ». Revue Internationale de Criminologie et de Police Technique et Scientifique, n³, pp.287-304.

Yébouet, H. (2011). « La lutte contre le racket policier en Côte d'Ivoire : Etat des lieux ». Revue Internationale de Criminologie et de Police Technique et Scientifique, $\mathrm{n}^{\circ} 1$, pp.33-48.

Yébouet, H. (2012). "Les dérives policières, une mise en cause de l'institution policière ». Revue Internationale de Criminologie et de Police Technique et Scientifique, $n^{\circ} 4$, pp.418-433.

\section{Journaux}

Fraternité - Matin du 26 Février 1999, p.6.

Fraternité - Matin du 18 et 19 Juillet 1998, p.6.

Jeune Afrique Economie, n²50 du 20/10/ au 02/11/ 1997 : “ Côte d'Ivoire : une police pour les policiers ».

L’Evénement du Jeudi, n487, du 3 au 9 Mars 1994: « Des ripoux dans la police ». p.32

Notre Voie du 29/07/2014, "Axe routier Abengourou - Ehuasso: 12 barrages à racket... »

Xinhua du 5 juillet 2014 : "Côte d'Ivoire : des mesures d'envergure pour juguler le racket ».

\section{Vidéocassettes / DVD}

Bah, S. (Producteur Délégué), Quassi, A. (Scénario/Dialogue), Soro, B. (Image), Mobio, G. (Son), Ouattara, Y. (Lumière) \& Bah, S. (Montage et mixage) (2005). Kemet Image, Lexys Films, Les Aventures du Sergent Digbeu 1, 2 et 3.

Diarrassouba, I. \& Baddy, A. (Producteurs Exécutifs), Guikou, A. (Réalisateur) Djep, B. (Directeur artistique), Mobio, G., Nogbou, J. (Son), Guikou, B.R. (Direction scénario) (2013). Côte Ouest Distribution DVD://, Production (Akwaba Films): Broutage.com 1, Internet est un outil de communication et non d'arnaque. La cybercriminalité est un délit.

Rocco \& Xavier (Producteurs Exécutifs), Guenaman, S. (Directeur de production), Kofi, F. (Scénariste), Lengani, E. H. (Réalisateur) et Touré, A. (Montage) (2009). ADJA-CI (Inter). Studios 225 Productions : Les Gaous d’Abidjan : Le Villageois 1 \& 2. 